\title{
Photopatternable Magnetic Hollowbots by Nd-Fe-B Nanocomposite for Potential Targeted Drug Delivery Applications
}

\author{
Hui Li *, Jing Chen, Jinjie Zhang, Jingyong Zhang, Guoru Zhao and Lei Wang * \\ Institute of Biomedical \& Health Engineering, Shenzhen Institutes of Advanced Technology, \\ Chinese Academy of Sciences, Shenzhen 518055, China; jing.chen@siat.ac.cn (J.C.); \\ jj.zhang@siat.ac.cn (J.Z.); jy.zhang@siat.ac.cn (J.Z.); gr.zhao@siat.ac.cn (G.Z.) \\ * Correspondence: hui.li1@siat.ac.cn (H.L.); wang.lei@siat.ac.cn (L.W.); \\ Tel.: +755-86392298 (H.L.); +755-86392298 (L.W.)
}

Received: 23 January 2018; Accepted: 5 April 2018; Published: 13 April 2018

check for updates

\begin{abstract}
In contrast to traditional drug administration, targeted drug delivery can prolong, localize, target and have a protected drug interaction with the diseased tissue. Drug delivery carriers, such as polymeric micelles, liposomes, dendrimers, nanotubes, and so on, are hard to scale-up, costly, and have short shelf life. Here we show the novel fabrication and characterization of photopatternable magnetic hollow microrobots that can potentially be utilized in microfluidics and drug delivery applications. These magnetic hollowbots can be fabricated using standard ultraviolet (UV) lithography with low cost and easily accessible equipment, which results in them being easy to scale up, and inexpensive to fabricate. Contact-free actuation of freestanding magnetic hollowbots were demonstrated by using an applied $900 \mathrm{G}$ external magnetic field to achieve the movement control in an aqueous environment. According to the movement clip, the average speed of the magnetic hollowbots was estimated to be $1.9 \mathrm{~mm} / \mathrm{s}$.
\end{abstract}

Keywords: microfabrication; photopatternable; magnetic composite; hollowbots; drug delivery

\section{Introduction}

In the past decades, there were normally a couple of routes of the drug delivery system: oral, transdermal, inhalation and injection. Following the rapid development of nanomaterials, micro-nanotechnology, biomedical technology, polymer science, physical chemistry, and so on, a targeted drug delivery system was proposed [1].

A targeted drug delivery system can prolong, localize, target and have a protected drug interaction with the diseased tissue. There are various types of drug delivery vehicles, such as polymeric micelles, liposomes, dendrimers, nanotubes, and microstrucutures, and so on. Due to their small size, high solubility and simple sterilization, polymeric micelles [2] are considered to be an ideal carrier for poorly water soluble drugs. But the physical stability of this carrier is a critical issue of polymeric micelles being microcarriers. Liposomes [3] are one of the most widely studied targeted delivery system, providing advantages such as low immunogenicity, biocompatibility, cell specificity and drug protection. On the other hand, there are also shortcomings such as poor scale-up, cost, short shelf life, and in some cases toxicity and off target effects. Due to their nanoscopic size, narrow polydispersity index, excellent control over molecular structure, availability of multiple functional groups at the periphery and cavities, dendrimers [4] have great potential in delivery carriers. But the high manufacturing cost, safety, toxicity and efficacy issues of dendrimers is still preventing them from clinical applications. Nanoparticles are a great alternative for drug delivery, such as polymer-iron 
oxide nanocomposites for enhanced permeability and retention (EPR)-independent delivery [5]. Microstructures [6], such as microneedles [7], micropumps [8], microswimmers [9], and so on, have been demonstrated as drug delivery carriers in recent researche; however, these kinds of structures are limited in loaded drug physical state, target location, and loading capability. Some recent magnetic micro-/nano drug delivery systems are summarized in Figure 1.

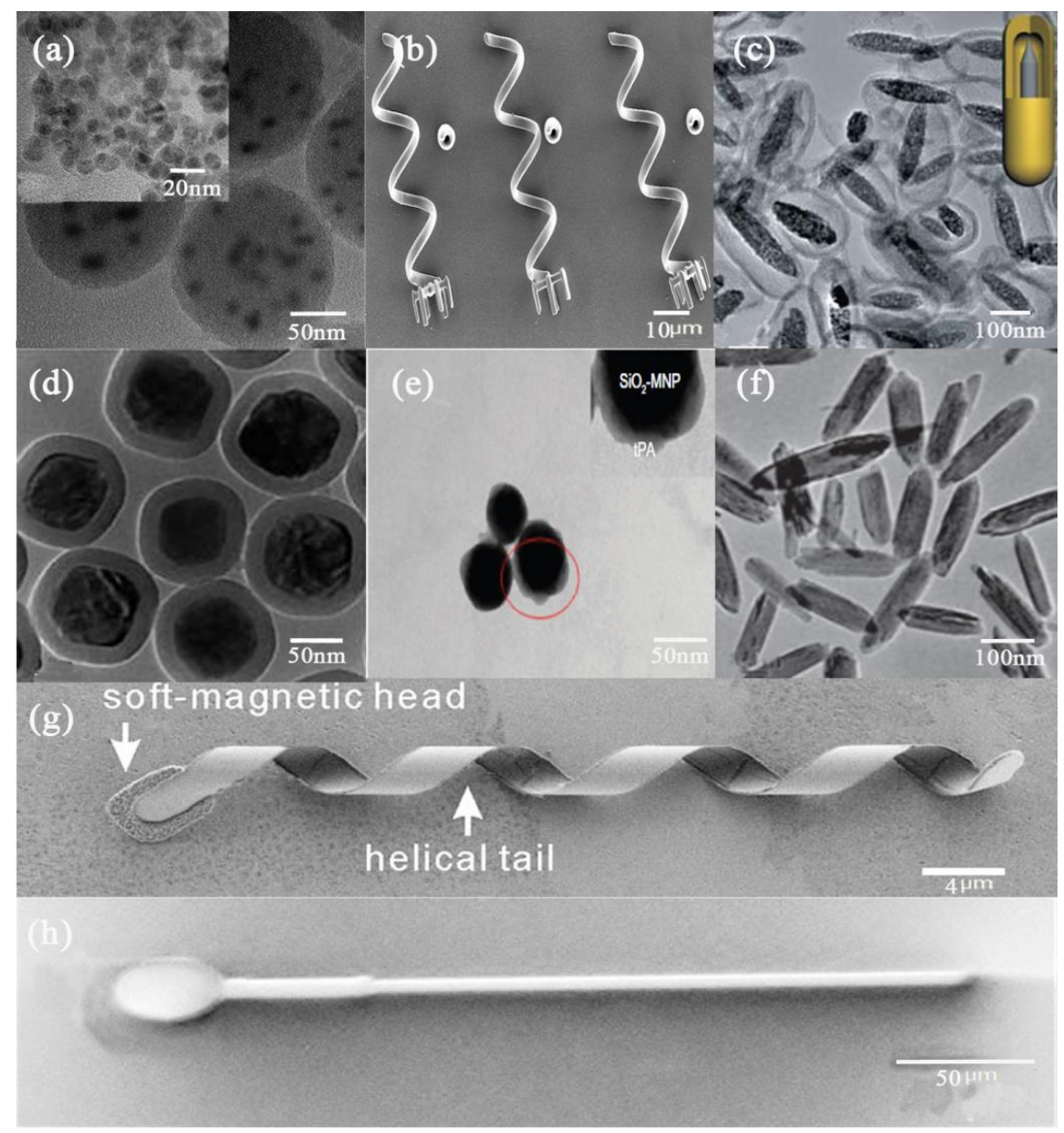

Figure 1. Recent magnetic micro-/nano- drug delivery systems. (a) The cyclo(Arg-Gly-Asp-D)doxorubicin/verapamil-magnetic nanoparticles-Poly(lactic acid-coglycolic acid) nanoparticles. Reproduced with permission from [10]. Copyright 2013, Elsevier; (b) An array of helical micromachines. Reproduced with permission from [9]. Copyright 2012, John Wiley and Sons; (c) Heterogeneous rattle-type $\mathrm{Fe}_{3} \mathrm{O}_{4} @ \mathrm{mSiO}_{2}$ nanoparticles. Reproduced with permission from [11]. Copyright 2011, The Royal Society of Chemistry; (d) Rattle-type magnetic mesoporous silica nanospheres. Reproduced with permission from [12], Copyright 2011, The Royal Society of Chemistry; (e) Tissue plasminogen activator bound to silica-coated magnetic nanoparticle after staining with phosphotungstic acid. Reproduced with permission from [13]; (f) Hollow core, magnetic, and mesoporous double-shell nanostructures. Reproduced with permission from [14]. Copyright 2011, John Wiley and Sons; (g) An untethered artificial bacterial flagella. Reproduced with permission from [15]. Copyright 2009, AIP Publishing.; (h) MagnetoSperm moving under the influence of the oscillating weak magnetic fields. Reproduced with permission from [16]. Copyright 2014, AIP Publishing.

Magnetic forces offer an attractive option for actuation in microelectromechanical systems (MEMS) and microscale systems because they scale favorably at micro- and nano-scale lengths [17,18]. The contact-free nature of magnetic actuation makes it ideal for applications where contamination must be avoided, such as interactions with cells or other biological samples [19], or where connecting 
the power source to the actuator would be cumbersome, such as freestanding microrobots [20]. And, unlike systems based on electrostatic or dielectric forces, magnetic actuators can operate in liquid or gas and are unaffected by the ionic concentration of the surrounding medium.

The benefits of microscale magnetic actuation have led to its implementation in a variety of MEMS and microfluidics devices, performing tasks such as wireless microfluidic mixing [21] and pumping of fluids [22]; in microrobotics, the use of magnetic force to provide wireless control and power is particularly appealing as it does not require the robots to be operated on a specialized surface and it can be used to perform complex three-dimensional motions [23]. Remotely-controlled microscale robots show potential in a variety of applications, including interaction with samples in lab-on-a-chip systems, navigation [16], in vivo delivery of cancer therapies, and performance of retinal- and neuro-surgical procedures [24]. But for many of these applications, ferro- or paramagnetic components are challenging to integrate into existing fabrication schemes.

Researchers have demonstrated various methods for creating magnetic microstructures, most of which can be characterized as either additive, subtractive, or polymer mixing methods. Deposition of magnetic materials via sputtering or evaporation [25] to achieve the microstructure magnetic. These methods could result in good purity of magnetic materials for small samples, but they are expensive and time-consuming for large ones. Etching and cutting [26] are subtractive methods to obtain magnetic microstructures. These methods can achieve good density of magnetic materials, but lack in precision. Polymer mixing [27] is a promising method for magnetic microstructure fabrication, but are expensive for many applications.

Our work builds on the Nd-Fe-B magnetic composite prepared and characterized in previous publications [28]. The paper will show the schematic of the microfabrication method for creating magnetic hollowbots in two geometries, and will also report on characterizations which provide control guidelines for actuating the hollowbots in aqueous environments. The method reported here differs from liposome, nanoparticles, micelle, nanotubes, and so on, used in previous works for loading and delivering drugs; in fabricating hollow microstructures as carriers for drug delivery systems in a specific method of using traditional lithography; and in the structures of the carriers and their materials, geometries, capacity and loading molecule within the hollowbots.

\section{Materials and Methods}

\subsection{Magnetic Composite Preparation}

The magnetic composite materials were made using Nd-Fe-B particles with $2 \mu \mathrm{m}$ average diameter (Magnequench MQFP-B-20076-084, Molycorp Inc., Greenwood Village, CO, USA) and SU-8 negative photoresist (MicroChem Corp., Westborough, MA, USA) with $4500 \mathrm{~mm}^{2} / \mathrm{s}$ kinematic viscosity. Composites were prepared by mixing $40 \%$ concentration $\mathrm{Nd}-\mathrm{Fe}-\mathrm{B}$ powder into SU 8 by mass fraction in microcentrifuge tubes. To attain a uniform dispersion and avoid settling of the magnetic particles, samples were mixed by vortexing (Vortex Genie 2, Scientific Industries, Inc., Bohemia, NY, USA) at $3000 \mathrm{rpm}$ for $45 \mathrm{~min}$ immediately before use. Unless otherwise stated, particle concentrations are given by fractional mass percentage in the following text.

\subsection{Hollowbots Fabrication}

The microfabrication process for hollowbots is a facile fabrication process for the construction of fully encapsulated and hollow microstructures using affordable and easy accessible patterned photolithographic processes. The basic idea is to construct a fully encapsulated space inside the microstructures using photopatternable layers and walls by stacking lithography. First, a sacrificial layer was spin-coated with Omnicoat (MicroChem) spin-coating at $500 \mathrm{rpm}$ for $5 \mathrm{~s}$, followed by $3000 \mathrm{rpm}$ for $30 \mathrm{~s}$, and baking at $200{ }^{\circ} \mathrm{C}$ for $1 \mathrm{~min}$, in accordance with the manufacturer's instructions. After the wafer cooled to room temperature, the bottom of the hollowbots was fabricated by spin-coating the mixed magnetic composite on the Omnicoat surface at $500 \mathrm{rpm}$ for $5 \mathrm{~s}$ (ramp rate of 
$100 \mathrm{rpm} / \mathrm{s}$ ) followed by $30 \mathrm{~s}$ at $4000 \mathrm{rpm}$ (ramp rate of $300 \mathrm{rpm} / \mathrm{s}$ ). This was followed by pre-exposure bake, UV exposure, and post-exposure bake that were all conducted according to the manufacturer's recommendations for pure SU-8, unless otherwise specified. Then another magnetic composite was applied onto the exposed layer and UV patterned following the same procedure for the first layer but exposed by a hollow rectangle to obtain the side walls of the hollowbots. On the other hand, the lid of the hollowbots was prepared on a cover slip with an Omnicoat sacrificial layer and a half pre-exposure baked magnetic composite layer following the same procedure as previously demonstrated. After development of the two exposed layers in SU-8 developer (MicroChem) with gentle agitation, the half pre-exposure baked lid was flipped and brought it into contact with the base structure, followed with pre-exposure bake, UV exposure through the transparent cover slip, curing the "lid" geometry while maintaining an internal void, and post-exposure bake. Detailed procedure flow is shown in Figure 2.

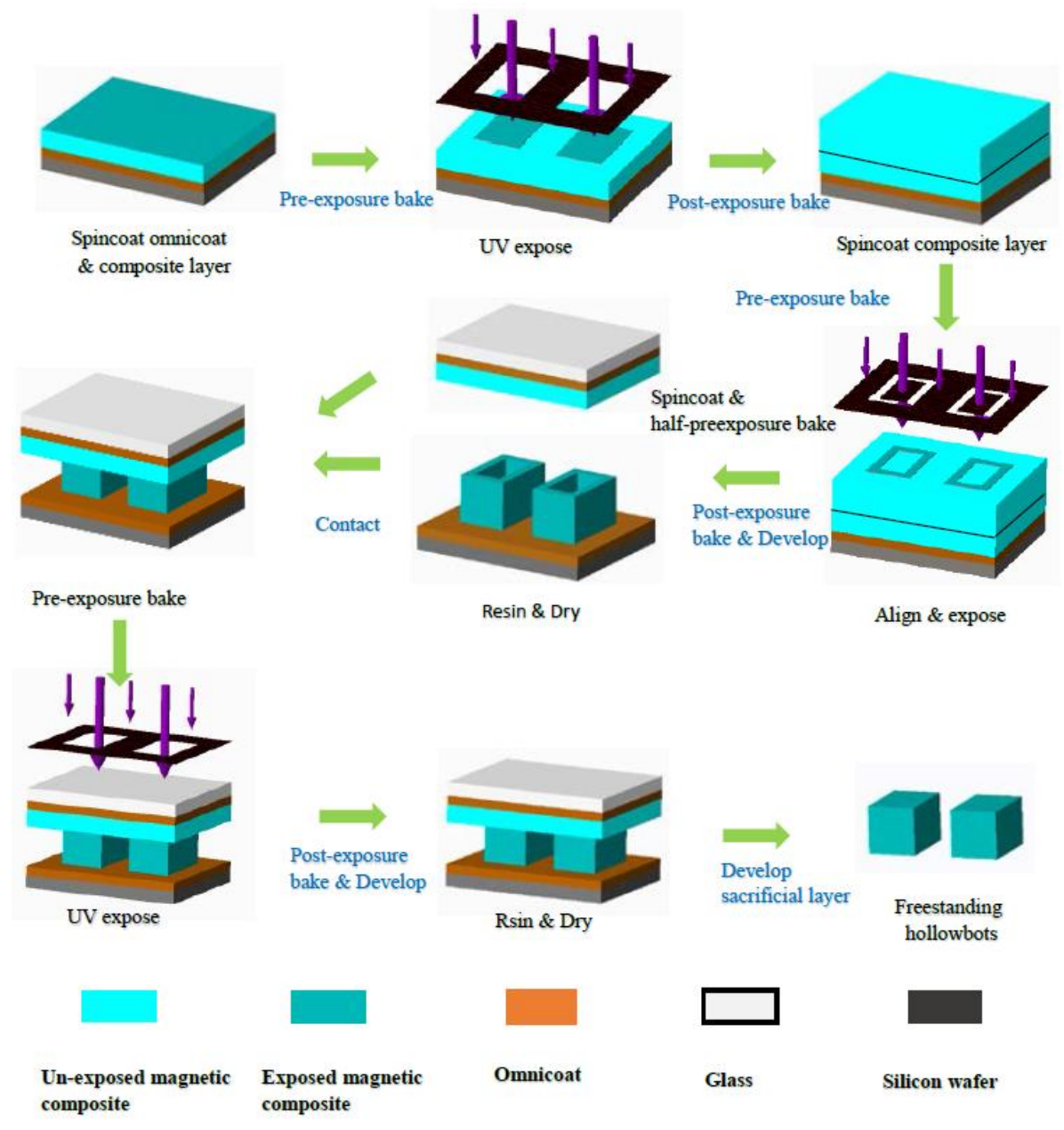

Figure 2. Schematic of magnetic hollowbots fabrication.

Freestanding magnetic hollowbots were released from the silicon surface and cover slip by chemically dissolving the sacrificial Omnicoat layer by soaking in PG Remover (MicroChem) for up to 45 min under gentle agitation. Individual robots were then carefully separated out using a micromanipulator. Alternatively, the PG Remover solution containing the released microrobots was pipetted into a microcentrifuge tube where a permanent magnet was used to collect the robots to the side of the vial; several wash steps were performed to remove the PG Remover and replace it with isopropyl alcohol for long-term storage. Scanning electron microscope (SEM) images of cylindrical and cuboid magnetic hollowbots are shown in Figure 3. 


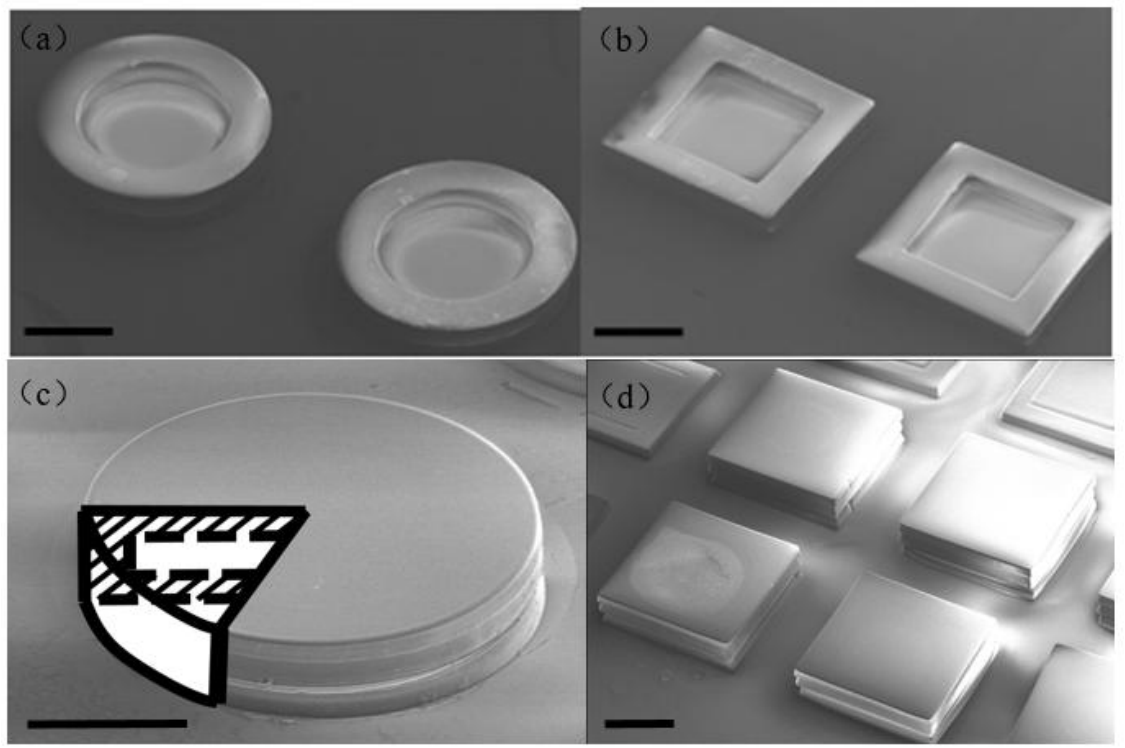

Figure 3. Scanning electron microscope (SEM) images of cylindrical (a) and cuboid (b) magnetic hollowbots before photopatterning top layer. (c) SEM image and sketch of the photopatterned magnetic cylindrical hollowbot structure. (d) SEM image of the photopatterned magnetic cuboid hollowbots. Scale bars $=100 \mu \mathrm{m}$.

\section{Characterization}

To obtain the optimal magnetic actuation performance of these hollowbots, uniform distribution of magnetic particles in the hollowbots is important for obtaining homogeneous magnetic properties, which could be characterized by the method developed in our previous publication [17], analyzing the optical microscopy images of the magnetic composite produced at various particle concentrations and spin speeds. Images taken by Nikon ECLIPSE Ci-POL (Nikon Instruments, Tokyo, Japan), and digitally processed using ImageJ software (1.8.0, National Institutes of Health (NIH), Bethesda, MD, USA) to dice each $250 \mu \mathrm{m} \times 250 \mu \mathrm{m}$ cured magnetic composite area into $10 \mu \mathrm{m} \times 10 \mu \mathrm{m}$ subsets and then calculate the percentage of each subset occupied by magnetic particles (black part of the optical image); and there are 625 subsets. The average $\mu$ and standard deviation $\sigma$ of this value was calculated for set of processing parameters. As particle concentration increases, we would expect $\mu \rightarrow 100 \%$ and as material uniformity increases, we would expect $\sigma \rightarrow 0$. Optical images for $40 \%$ samples with 1000 and $4000 \mathrm{rpm}$ are shown in Figure 4. The statistical parameters, histograms of values for each sample are shown in Figure 5.

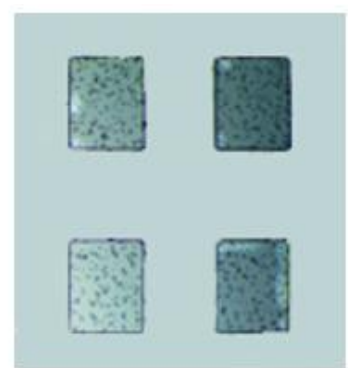

(a)

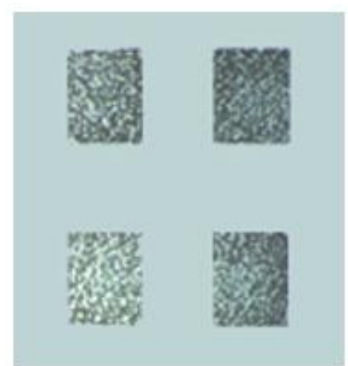

(b)

Figure 4. Optical images of (a) 40\%, $1000 \mathrm{rpm}$, (b) 40\%, $4000 \mathrm{rpm}$ samples. 

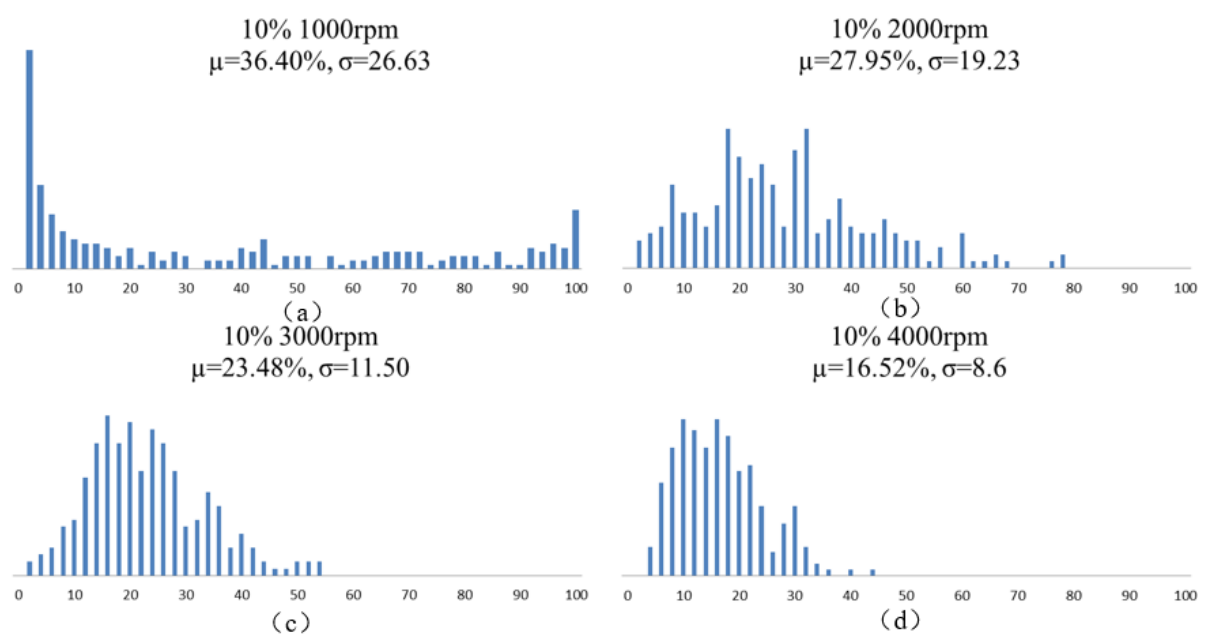

$40 \% 1000 \mathrm{rpm}$ $\mu=85.31 \%, \sigma=16.79$

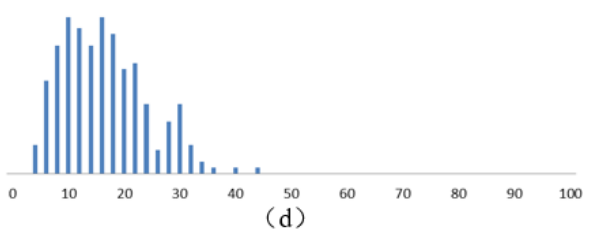

$40 \% 2000 \mathrm{rpm}$

$\mu=75.71 \%, \sigma=10.36$
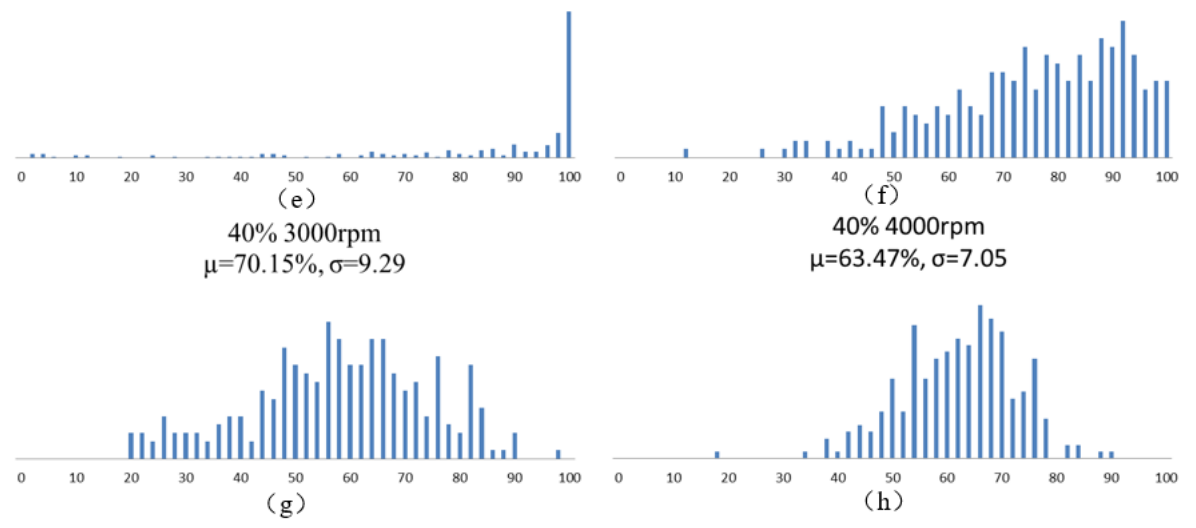

Figure 5. (a-d) 10\% mass fraction Nd-Fe-B composite uniformity for different fabrication spincoating speed. Histograms represent for samples at given processing parameters. (e-h) $40 \%$ mass fraction $\mathrm{Nd}-\mathrm{Fe}-\mathrm{B}$ composite uniformity for different fabrication spincoating speed. Histograms represent for samples at given processing parameters.

The experiment data analysis has shown that the Nd-Fe-B particles are favor to cluster together and create large pieces at lower spincoat speed; while at higher speed, it is much easy to distribute the magnetic composite uniformly. However, it also decreases the microstructure thickness. Magnetic structure fabricated at a higher spin speed will have fewer magnetic particles included; therefore, to create hollowbots for magnetic actuation, each deposited layer would be better to spincoat at high speed with good particle distribution and high particle count, multiple layers of the magnetic composite can be deposited sequentially on a silicon wafer and patterned with UV exposure to achieve the wanted thickness. A microfluidic testing was carried out to confirm the quality of bonding and the leakage of the hollowbots chamber. The test consisted of inserting an ink into the chamber and the hollowbots could withstand pressure up to $400 \mathrm{kPa}$ without any liquid leakage.

\section{Actuation}

Freestanding hollowbots were obtained by releasing from the wafer and coverslip as described in previous session and transferred with gentle pipetting to a glass dish filled with isopropyl alcohol. With trapped air in the chamber, hollowbots could float in the isopropyl alcohol environment. An external magnetic field was chosen to actuate the Nd-Fe-B hollowbots, which was generated by a permanent magnet (Nd-Fe-B 56, $12 \mathrm{~mm} \times 12 \mathrm{~mm} \times 12 \mathrm{~mm}$, K\&J Magnetics, Inc., Pipersville, PA, USA) with surface field strength of $5600 \mathrm{G}$ held at a distance of $10 \mathrm{~mm}$ from the hollowbot. At this 
distance, the strength of the magnetic field was approximately $900 \mathrm{G}$, which is able to overcome surface friction, adhesion forces and the drag force applied on the microrobots. The movement of the permanent magnet was held by hand and used to guide the hollowbot move. Using this method, we were able to successfully guide magnetic hollowbots at an average speed of $1.9 \mathrm{~mm} / \mathrm{s}$, estimated from the movement clip. The captured images from the magnetic hollowbots actuation clip are shown in Figure 6.

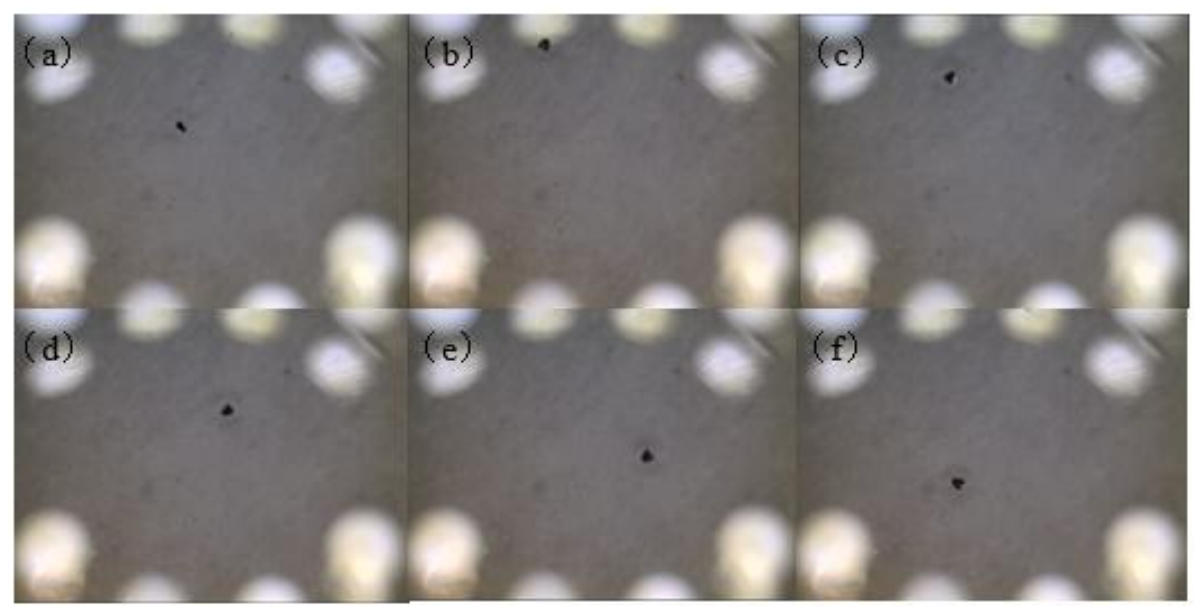

Figure 6. Captured images (every $2 \mathrm{~s}$ ) from the clip of hollowbots actuated by external magnetic field in isopropyl alcohol. (a) $0 \mathrm{~s}$; (b) $2 \mathrm{~s}$; (c) $4 \mathrm{~s}$; (d) $6 \mathrm{~s}$; (e) $8 \mathrm{~s}$; (f) $10 \mathrm{~s}$.

\section{Conclusions}

In this paper, a facile microfabrication of magnetic Nd-Fe-B hollow microrobots was developed by depositing magnetic composite sequentially and patterning the bottom and the side walls of the hollowbots. Contacting with the prepared composite walls on the coverslip and photopatterning the top layer, the magnetic hollowbots are created. In characterization of the uniform distribution with spin speed and particle concentration, it was found that particles could be uniformly dispersed within the polymer matrix by using high spin speed during fabrication without requiring chemical modification of either of the two composite components. The contact-free actuation of the magnetic microrobots was also demonstrated by moving a freestanding hollowbots in an aqueous environment with an external magnetic field. According to the clip, the average speed of the hollowbots could reach $1.9 \mathrm{~mm} / \mathrm{s}$. The composite magnetic hollowbots demonstrated here can be applied in a variety of applications where wireless actuation is necessary, including microfluidics, microrobotics, and drug delivery.

Acknowledgments: This technology was partially supported by Guangdong Natural Science Foundation (Grant NO. 2017A030310469), Shenzhen Fundamental Research Project (JCYJ20170307165039508), Key deployment project of Chinese Academy of Sciences (Grant No. KFZD-SW-214), the Technology Innovation Fund of Shenzhen Peacock Talent (KQJSCX2016030114125686), and SIAT Innovation Program for Excellent Young Researchers. (Grant No. 2016053).

Author Contributions: Hui Li and Lei Wang conceived and designed the experiments; Hui Li performed the experiments; Hui Li and Jingyong Zhang analyzed the data; Jing Chen contributed reagents/materials/analysis tools; Jinjie Zhang edited the images; Jing Chen prepared the copyright files; Hui Li, Jinjie Zhang and Guoru Zhao wrote the paper.

Conflicts of Interest: The authors declare no conflict of interest.

\section{References}

1. Allen, T.M.; Cullis, P.R. Drug delivery systems: Entering the mainstream. Science 2004, 303, 1818-1822. [CrossRef] [PubMed] 
2. Talelli, M.; Barz, M.; Rijcken, C.J.; Kiessling, F.; Hennink, W.E.; Lammers, T. Core-crosslinked polymeric micelles: Principles, preparation, biomedical applications and clinical translation. Nano Today 2015, 10, 93-117. [CrossRef] [PubMed]

3. Blanco, E.; Shen, H.; Ferrari, M. Principles of nanoparticle design for overcoming biological barriers to drug delivery. Nat. Biotechnol. 2015, 33, 941-951. [CrossRef] [PubMed]

4. Caminade, A.-M.; Turrin, C.-O. Dendrimers for drug delivery. J. Mater. Chem. B 2014, 2, 4055-4066. [CrossRef]

5. Park, J.; Kadasala, N.R.; Abouelmagd, S.A.; Castanares, M.A.; Collins, D.S.; Wei, A.; Yeo, Y. Polymer-iron oxide composite nanoparticles for epr-independent drug delivery. Biomaterials 2016, 101, 285-295. [CrossRef] [PubMed]

6. McHugh, K.J.; Nguyen, T.D.; Linehan, A.R.; Yang, D.; Behrens, A.M.; Rose, S.; Tochka, Z.L.; Tzeng, S.Y.; Norman, J.J.; Anselmo, A.C.; et al. Fabrication of fillable microparticles and other complex 3d microstructures. Science 2017, 357, 1138-1142. [CrossRef] [PubMed]

7. Traverso, G.; Schoellhammer, C.M.; Schroeder, A.; Maa, R.; Lauwers, G.Y.; Polat, B.E.; Anderson, D.G.; Blankschtein, D.; Langer, R. Microneedles for drug delivery via the gastrointestinal tract. J. Pharm. Sci. 2015, 104, 362-367. [CrossRef] [PubMed]

8. Tandon, V.; Kang, W.S.; Robbins, T.A.; Spencer, A.J.; Kim, E.S.; McKenna, M.J.; Kujawa, S.G.; Fiering, J.; Pararas, E.E.; Mescher, M.J.; et al. Microfabricated reciprocating micropump for intracochlear drug delivery with integrated drug/fluid storage and electronically controlled dosing. Lab Chip 2016, 16, 829-846. [CrossRef] [PubMed]

9. Tottori, S.; Zhang, L.; Qiu, F.; Krawczyk, K.K.; Franco-Obregón, A.; Nelson, B.J. Magnetic helical micromachines: Fabrication, controlled swimming, and cargo transport. Adv. Mater. 2012, 24, 811-816. [CrossRef] [PubMed]

10. Shen, J.-M.; Gao, F.-Y.; Yin, T.; Zhang, H.-X.; Ma, M.; Yang, Y.-J.; Yue, F. Crgd-functionalized polymeric magnetic nanoparticles as a dual-drug delivery system for safe targeted cancer therapy. Pharm. Res. 2013, 70, 102-115. [CrossRef] [PubMed]

11. He, Q.; Shi, J. Mesoporous silica nanoparticle based nano drug delivery systems: Synthesis, controlled drug release and delivery, pharmacokinetics and biocompatibility. J. Mater. Chem. 2011, 21, 5845-5855. [CrossRef]

12. Wu, H.; Liu, G.; Zhang, S.; Shi, J.; Zhang, L.; Chen, Y.; Chen, F.; Chen, H. Biocompatibility, mr imaging and targeted drug delivery of a rattle-type magnetic mesoporous silica nanosphere system conjugated with peg and cancer-cell-specific ligands. J. Mater. Chem. 2011, 21, 3037-3045. [CrossRef]

13. Chen, J.-P.; Yang, P.-C.; Ma, Y.-H.; Tu, S.-J.; Lu, Y.-J. Targeted delivery of tissue plasminogen activator by binding to silica-coated magnetic nanoparticle. Int. J. Nanomed. 2012, 7, 5137. [CrossRef] [PubMed]

14. Wu, H.; Zhang, S.; Zhang, J.; Liu, G.; Shi, J.; Zhang, L.; Cui, X.; Ruan, M.; He, Q.; Bu, W. A hollow-core, magnetic, and mesoporous double-shell nanostructure: In situ decomposition/reduction synthesis, bioimaging, and drug-delivery properties. Adv. Funct. Mater. 2011, 21, 1850-1862. [CrossRef]

15. Zhang, L.; Abbott, J.J.; Dong, L.; Kratochvil, B.E.; Bell, D.; Nelson, B.J. Artificial bacterial flagella: Fabrication and magnetic control. Appl. Phys. Lett. 2009, 94, 064107. [CrossRef]

16. Khalil, I.S.; Dijkslag, H.C.; Abelmann, L.; Misra, S. Magnetosperm: A microrobot that navigates using weak magnetic fields. Appl. Phys. Lett. 2014, 104, 223701. [CrossRef]

17. Trimmer, W.S.N. Microrobots and micromechanical systems. Sens. Actuator 1989, 19, 267-287. [CrossRef]

18. Cugat, O.; Delamare, J.; Reyne, G. Magnetic micro-actuators and systems (magmas). IEEE Trans. Magn. 2003, 39, 3607-3612. [CrossRef]

19. Steager, E.B.; Selman Sakar, M.; Magee, C.; Kennedy, M.; Cowley, A.; Kumar, V. Automated biomanipulation of single cells using magnetic microrobots. Int. J. Robot. Res. 2013, 32, 346-359. [CrossRef]

20. Li, H.; Zhang, J.; Zhang, N.; Kershaw, J.; Wang, L. Fabrication and wireless micromanipulation of magnetic biocompatible microrobots using microencapsulation for microrobotics and microfluidics applications. J. Microencapsul. 2016, 33, 712-717. [CrossRef] [PubMed]

21. Zhou, B.; Xu, W.; Syed, A.A.; Chau, Y.; Chen, L.; Chew, B.; Yassine, O.; Wu, X.; Gao, Y.; Zhang, J.; et al. Design and fabrication of magnetically functionalized flexible micropillar arrays for rapid and controllable microfluidic mixing. Lab Chip 2015, 15, 2125-2132. [CrossRef] [PubMed]

22. Said, M.M.; Yunas, J.; Pawinanto, R.E.; Majlis, B.Y.; Bais, B. Pdms based electromagnetic actuator membrane with embedded magnetic particles in polymer composite. Sens. Actuators A Phys. 2016, 245, 85-96. [CrossRef] 
23. Kummer, M.P.; Abbott, J.J.; Kratochvil, B.E.; Borer, R.; Sengul, A.; Nelson, B.J. Octomag: An electromagnetic system for 5-dof wireless micromanipulation. IEEE Trans. Robot. 2010, 26, 1006-1017. [CrossRef]

24. Chatzipirpiridis, G.; Ergeneman, O.; Pokki, J.; Ullrich, F.; Fusco, S.; Ortega, J.A.; Sivaraman, K.M.; Nelson, B.J.; Pané, S. Electroforming of implantable tubular magnetic microrobots for wireless ophthalmologic applications. Adv. Healthc. Mater. 2015, 4, 209-214. [CrossRef] [PubMed]

25. Basumatary, H.; Chelvane, J.A.; Rao, D.S.; Kamat, S.; Ranjan, R. Effect of sputtering parameters on the structure, microstructure and magnetic properties of tb-fe films. Thin Solid Films 2015, 583, 1-6. [CrossRef]

26. Jeon, M.H.; Yang, K.C.; Park, J.W.; Yun, D.H.; Kim, K.N.; Yeom, G.Y. Etching of magnetic tunnel junction materials using reactive ion beam. J. Nanosci. Nanotechnol. 2016, 16, 11823-11830. [CrossRef]

27. Prasad, B.B.; Jauhari, D. Double-ion imprinted polymer@ magnetic nanoparticles modified screen printed carbon electrode for simultaneous analysis of cerium and gadolinium ions. Anal. Chim. Acta 2015, 875, 83-91. [CrossRef] [PubMed]

28. Li, H. Fabrication of Magnetic Two-Dimensional and Three-Dimensional Microstructures for Microfluidics and Microrobotics Applications. Ph.D. Thesis, University of Kentucky, Lexington, KY, USA, 2014.

(C) 2018 by the authors. Licensee MDPI, Basel, Switzerland. This article is an open access article distributed under the terms and conditions of the Creative Commons Attribution (CC BY) license (http://creativecommons.org/licenses/by/4.0/). 